\title{
Review on Complete Blood Count Parameters of Animals - A Formidable Aid in Veterinary Disease Diagnosis
}

\author{
Harshit Saxena $^{1 *}$ and Shalvi Srivastava ${ }^{2}$ \\ ${ }^{1}$ Intern, CoVSc and AH, DUVASU, Mathura, India \\ ${ }^{2} U G$ Scholar, CoVSc and AH, DUVASU, Mathura, India \\ *Corresponding Author: Harshit Saxena, Intern (BVSc and AH), CoVSc and AH, \\ DUVASU, Mathura, India.
}

Received: September 14, 2021

Published: September 29, 2021

(C) All rights are reserved by Harshit Saxena and Shalvi Srivastava.

\begin{abstract}
Diagnosis of an ailment is soul of clinical practice and perhaps the biggest challenge as far as veterinary clinical practice is concerned. A long array of procedures, diagnostic test and methods are available for a clinician that can aid him to make his interpretation and make a promising opinion about any disease condition. Blood profiling remained one of the most valuable Diagnostic technique since times and in it Complete Blood Count/ Picture is very cardinal test that provide a glance about pathophysiological state of a subject in very quick convenient and economical way. Thus this review aims at understanding different parameters of Complete Blood Count (CBC) test in important farm and companion animals. It also give insights into modern technology that is making these tests more refined and easily accessible to patients as well as to clinicians.
\end{abstract}

Keywords: Complete Blood Count; Veterinary; Diagnosis; Erythrocytes; Leucocytes; Thrombocytes; Haematology

\section{Abbreviations}

CBC: Complete Blood Count; TEC: Total Erythrocytic Count; PCV: Packed Cell Volume; Hb/Hgb: Haemoglobin; MCV: Mean Corpuscular Volume; MCH: Mean Corpuscular Haemoglobin; MCHC: Mean Corpuscular Haemoglobin Concentration; TLC: Total Leucocyte Count; RDW: Red Cell Distribution Width; ESR: Erythrocyte Sedimentation Rate

\section{Introduction}

With the advent of emerging technologies, advanced medical devices and specialized and novel approaches, the healthcare industry is transforming. It is a well-established fact that hectic lifestyles have resulted in the ignorance of important steps in sustaining a healthier lifestyle. However, with the emergence of medical conditions that are exceptional and quite rare, early and correct diagnosis of the disorder is of utmost importance.
Since the patient has too much to lose if the diagnosis is incorrect, diagnosis is more critical than ever before. A diagnosis error could result in the patient being refused timely, appropriate treatment or being given potentially harmful, inaccurate medications. Whereas immediate care may have restored a patient's health, the effects of a misdiagnosis can be catastrophic. When a diagnosis is reliable and taken promptly, a patient has the highest chance of a better health result because professional decision making is geared to a correct view of the patient's health condition.

Five distinct types of diagnosis are utilized for accurate detection of the disorder [1]:

- Clinical: A clinical diagnosis is based on the symptoms both described by the patient and observed by the professional, rather than diagnostic tests [1]. 
- Laboratory: Laboratory diagnosis is based on a cellular examination. Laboratory tests may identify organisms directly (visually, using a microscope, growing the organism in culture) or indirectly (identifying antibodies to the organism). This includes analysis of fluid samples, tissue samples, microscopy, culture techniques and other data from long-term patient observation $[1,2]$.

- Radiology: Diagnostic radiology is the branch of medicine that employs non-invasive imaging scans to identify patients. Low doses of radiation are often used in experiments and instruments to produce extremely accurate photographs of an environment. For example- Radiography (X-rays), Ultrasound, Computed Tomography (CT) Scans, Magnetic Resonance Imaging (MRI) Scans, Nuclear Medicine Scans [1].

- Differential: Differential diagnosis is a process wherein a doctor differentiates between two or more conditions that could be behind a person's symptoms. A differential diagnostic approach is helpful when there may be multiple potential causes to consider $[1,3]$.

- Nursing: A nursing diagnosis is similar to clinical diagnoses. The underlying differences being the inclusion of moment-tomoment patient interaction and knowledge. Lastly, a nursing diagnosis has a focus on how a patient responds to treatment [1].

In this age of high-tech diagnostic tools like Radiologic technology and Nuclear Magnetic Resonance (NMR), one basic test the complete blood count (CBC) remains at the centre. Millions of different cells are present in a single drop of blood, and a veterinarian will analyse and diagnose a wide range of conditions; from anaemia and autoimmune diseases to cancers and infections just by looking at half a spoon of blood from the patient.

A complete blood count refers to the count of the total number of cells in a certain amount of blood, including red blood cells (erythrocytes), white blood cells (leukocytes) and platelets (thrombocytes). It also provides relevant information about haemoglobin and other plasma proteins. This test is usually called CBC and is one of the most common tests to check for abnormalities in the blood which directly mirror the health status of the animal.
Blood collection sites in different species

\begin{tabular}{|c|c|}
\hline [4] Species & Site of collection \\
\hline $\begin{array}{c}\text { Cattle, buffalo, sheep, goat, } \\
\text { horse \& large wild ruminants }\end{array}$ & Jugular vein \\
\hline Dog & $\begin{array}{c}\text { Cephalic/Femoral/Saphenous/ } \\
\text { Jugular/Tibial vein }\end{array}$ \\
\hline Cat & $\begin{array}{c}\text { Ear/Femoral/Cephalic/Jugular/ } \\
\text { Saphenous vein }\end{array}$ \\
\hline Pig & Anterior vena cava/Ear vein \\
\hline Birds & Wing/Comb vein/Heart \\
\hline Rabbit/Guinea pigs & Heart/Ear vein \\
\hline Rat/mouse & Heart/Orbital sinus (Inner \\
& canthus) \\
\hline
\end{tabular}

Table 1

\section{Blood parameters}

Erythrocytes (RBC)

\section{Total erythrocytic count (TEC)}

TEC is defined as the number of erythrocytes present in a given volume of blood. Erythrocytes are most numerous blood cells and are generally represented in millions $\left(10^{6}\right)$ cells per $\mu \mathrm{l}$ (microlitre) of blood. ( $1 \mu \mathrm{l}=10^{-6}$ litre $)$.

The normal values of TEC in different species are:

\begin{tabular}{|c|c|}
\hline [5] Species & TEC $($ million $/ \boldsymbol{\mu l})$ \\
\hline Cattle/Buffalo & $5-10$ \\
\hline Horse & $6-10.4$ \\
\hline Sheep & $9-15$ \\
\hline Goat & $8-18$ \\
\hline Dog & $4.95-7.87$ \\
\hline Cat & $5-10$ \\
\hline Pig & $5-8$ \\
\hline Poultry & 1.7 \\
\hline
\end{tabular}

Table 2

\section{Haemoglobin (Hb) or (Hgb)}

This test measures the amount of haemoglobin present in 100 $\mathrm{ml}$ of blood, and is expressed in gram percent or gm/dl. 
The normal values of $\mathrm{Hb}$ in different species are:

\begin{tabular}{|c|c|}
\hline [5] Species & Hb(gm\%) \\
\hline Cattle/Buffalo & $8-15$ \\
\hline Horse & $10.1-16.1$ \\
\hline Sheep & $9-15$ \\
\hline Goat & $8-18$ \\
\hline Dog & $11.9-18.9$ \\
\hline Cat & $9.8-15.4$ \\
\hline Pig & $10-16$ \\
\hline Poultry & 12.2 \\
\hline
\end{tabular}

Table 3

\section{Packed cell volume (PCV)}

It is the volume of packed erythrocyte (on centrifugation) per hundred volumes of blood. It is expressed in percentage (\%).

PCV in different species.

\begin{tabular}{|c|c|}
\hline [5] Species & PCV (\%) \\
\hline Cattle/Buffalo & $24-46$ \\
\hline Horse & $27-43$ \\
\hline Sheep & $27-45$ \\
\hline Goat & $22-38$ \\
\hline Dog & $35-57$ \\
\hline Cat & $30-45$ \\
\hline Pig & $36-43$ \\
\hline Poultry & 32 \\
\hline
\end{tabular}

Table 4

\section{Mean corpuscular volume or MCV}

MCV is the average volume of red cells in a specimen. It is expressed in femtolitre (fL).

\begin{tabular}{|c|c|}
\hline [5] Species & MCV (fL) \\
\hline Cattle/Buffalo & $40-60$ \\
\hline Horse & $37-49$ \\
\hline Sheep & $28-40$ \\
\hline Goat & $16-25$ \\
\hline Dog & $66-77$ \\
\hline Cat & $39-55$ \\
\hline Pig & $52-62$ \\
\hline Poultry & 174 \\
\hline
\end{tabular}

Table 5
Mean corpuscular haemoglobin or $\mathrm{MCH}$

An $\mathrm{MCH}$ value refers to the average quantity of haemoglobin present in a single red blood cell [6].

\begin{tabular}{|c|c|}
\hline [5] Species & MCH (pg) \\
\hline Cattle/Buffalo & $11-17$ \\
\hline Horse & $13.7-18.2$ \\
\hline Sheep & $8-12$ \\
\hline Goat & $5.2-8$ \\
\hline Dog & $21-26.2$ \\
\hline Cat & $13-17$ \\
\hline Pig & $17-21$ \\
\hline Poultry & 61 \\
\hline
\end{tabular}

Table 6

Mean corpuscular haemoglobin concentration or MCHC

The MCHC is a measure of the concentration of haemoglobin in a given volume of packed red blood cell [7].

\begin{tabular}{|c|c|}
\hline [5] Species & MCHC(g/dl) \\
\hline Cattle/Buffalo & $30-36$ \\
\hline Horse & $35.3-39.3$ \\
\hline Sheep & $31-34$ \\
\hline Goat & $30-36$ \\
\hline Dog & $32-36.3$ \\
\hline Cat & $30-36$ \\
\hline Pig & $30-34$ \\
\hline Poultry & 33 \\
\hline
\end{tabular}

Table 7

RDW

A red cell distribution width (RDW) test is a measurement of the range in the volume and size of your red blood cells (erythrocytes) [8].

\begin{tabular}{|c|c|c|}
\hline Species & RDWCV & RDWSD \\
\hline Horse & $17.8 \%$ & $22.08 \%$ \\
\hline Dog & $13.8 \%$ & $23.61 \%$ \\
\hline Cat & $25.2 \%$ & $20.40 \%$ \\
\hline
\end{tabular}

Table 8 
Disorders related to deviation in normal erythrocytic parameters

A deviation from the normal level of these parameters can indicate a number of disorders like.

\section{Erythrocytosis}

- It is an increment in the concentration of $\mathrm{RBC}$ count, $\mathrm{Hb}$ concentration and packed cell volume [9].

- It can be relative or absolute in nature.

\section{Relative erythrocytosis}

Relative erythrocytosis occurs from dehydration or haemoconcentration. Although the haematocrit and erythrocyte numbers are increased, the total erythrocyte mass is normal. Plasma proteins are affected by shifts in fluid volume in the same manner as erythrocytes. Estimation of the haematocrit or packed cell volume and plasma proteins gives some analysis of the degree of dehydration [9].

\section{Absolute erythrocytosis}

This is an actual increase in erythrocyte number in blood. It can be primary erythrocytosis or secondary erythrocytosis [9]:

- Primary Erythrocytosis: [9] It occurs from proliferation of marrow erythroid precursors which are no longer influenced by erythropoietin. [10] It occurs as a result of polycythaemia vera, a myeloproliferative neoplasm in which abnormal cells in the bone marrow produce too many red blood cells along with excessive numbers of white blood cells and platelets. Occasionally, only red blood cell production is increased. Although this condition is quite uncommon, some cases have been reported in cats and dogs.

- Secondary Erythrocytosis: [9] This condition occurs from excessive production of erythropoietin as a response to hypoxaemia (insufficient oxygenation in blood). The increased viscosity of the blood causes impairment in blood flow, cyanosis, congestion and increased tendency for thrombosis. This is seen in chronic lung and heart diseases, renal tumour, renal cyst and cobalt toxicity.
Disorders and conditions that cause erythrocytosis are [19]:

- Haemoconcentration:

- Dehydration

- $\quad$ Endotoxic shock

- $\quad$ Splenic contraction.

- Secondary appropriate erythrocytic disorders:

- $\quad$ Right-to-left shunts, congenital or acquired

- $\quad$ Chronic pulmonary disease

- Hyperthyroidism.

- Secondary inappropriate erythrocytic disorders:

- Renal neoplasms, cysts or diseases

- $\quad$ Other neoplasm (hepatoma).

- Primary erythrocytic disorders:

- $\quad$ Primary erythrocytosis

- $\quad$ Polycythaemia vera (polycythaemia rubra vera).

- Idiopathic erythrocytosis.

\section{Oligocythaemia}

This refers to decreased levels of TEC, Hb, PCV in the blood. It can be relative or absolute in nature [9].

\section{Relative oligocythaemia}

In this condition, there is an increase in the blood volume (haemodilution) with normal number of erythrocytes, but the number of RBCs per unit blood decreases.

\section{Absolute oligocythaemia}

This refers to a decrease in the total number of erythrocytes present in the blood. 


\section{Anaemia}

It is the reduction in oxygen carrying capacity of the blood and can be result of oligocythaemia (reduction in RBC count); or due to less concentration of $\mathrm{Hb}$ in blood or due to reduction in total blood volume generally seen in cases of dehydration, or excessive blood loss from injury or haemorrhage [9].

Anaemia can be regenerative or non-regenerative depending on the presence or absence of compensatory erythropoiesis.

\section{Regenerative anaemia}

In a regenerative anaemia, the bone marrow responds appropriately to the decreased number of red blood cells by increasing production of new blood cells. This type of anaemia occurs from haemorrhage or haemolysis

\section{Haemorrhagic anaemia}

Anaemia caused due to sudden loss of blood due to an accident/ injury. This can occur in parasitic diseases such as bovine pediculosis, severe flea infestations of puppies and kittens, and hookworms in puppies. Chronic immune-mediated thrombocytopaenia in dogs and gastrointestinal neoplasms in older animals may also cause iron deficiency due to chronic extra-corporeal haemorrhage.

\section{Haemolytic anaemia}

Anaemia caused due to haemolysis (rupture or burst of RBC) within the circulatory system. The haemolysis can be due to certain toxins or due to congenital conditions like Sickle cell anaemia.

Disorders and conditions that cause haemolytic anaemia are [19]:

- Immune haemolytic disorders:

- Idiopathic (includes autoimmune)

- $\quad$ Drug induced

- $\quad$ Vaccine associated.

- Alloimmune:

- Neonatal isoerythrolysis

- $\quad$ Blood transfusion reactions.
- Haemolysis induced by bacterial and viral infections:

- $\quad$ Mycoplasma spp.

- $\quad$ Anaplasma spp.

- $\quad$ Leptospira spp.

- Clostridium spp. causing erythrocyte membrane damage by phospholipases:

- Bacillary haemoglobinuria (Clostridium haemolyticum or C. novyii)

- Yellow lamb disease (Clostridium perfringens, type A)

- Clostridial infections in horses

- $\quad$ EIAV

- $\quad$ FeLV

- Haemolysis associated with other infection (e.g., Ehrlichial)

- $\quad$ Erythrocytic metabolic defects (acquired or inherited):

- Oxidative damage:

- Heinz body haemolysis

- $\quad$ Eccentrocytic haemolysis (acquired or inherited).

- Defects in ATP generation:

- $\quad$ Pyruvate kinase deficiency

- Phosphofructokinase deficiency

- Hypophosphatemic haemolysis

- L-sorbose intoxication.

- Defects in heme synthesis that result in porphyria:

- Bovine congenital erythropoietic porphyria

- $\quad$ Feline erythropoietic porphyria.

- Erythrocyte fragmentation in blood creating schizocytes, keratocytes, acanthocytes:

Citation: Harshit Saxena and Shalvi Srivastava. "Review on Complete Blood Count Parameters of Animals - A Formidable Aid in Veterinary Disease Diagnosis". Acta Scientific Veterinary Sciences 3.10 (2021): 80-96. 
- Intra vascular coagulation (localised or disseminated)

- $\quad$ Vasculitis

- Hemangiosarcoma.

- $\quad$ Rheologic processes:

- Caval syndrome of dirofilariasis

- $\quad$ Cardiac valvular disease.

- Haemolytic anaemia of other or unknown pathogeneses:

- Protozoal infections:

- $\quad$ Babesia spp.

- $\quad$ Theileria spp.

- Trypanosoma spp.

- Heparin induced haemolysis

- Iatrogenic hypo-osmolar haemolysis

- $\quad$ Envenomation (snakes, spiders, insects)

- Hemophagocytic histiocytic sarcoma

- Idiopathic nonspherocytic haemolytic disorders with increased osmotic fragility

- Hereditary nonspherocytic haemolytic anaemia of beagles

- Idiopathic haemolytic anaemia of Abyssinian and Somali cats

- Haemolytic syndrome in horses with liver disease.

\section{Non-regenerative anaemia}

In a nonregenerative anaemia, the bone marrow responds inadequately to the increased need for red blood cells.

\section{Deficiency anaemia}

Anaemia caused to deficiency of any of the important components required for RBC or Hb synthesis like- Iron, Copper, Vitamin $\mathrm{B}_{12}$, etc [9].

\section{Aplastic anaemia}

Anaemia caused by the absence or slow rate of RBC production, due to drugs or exposure to radiation (X-rays, $y$ rays) [9].

\section{Anaemia of chronic diseases}

The anaemia of chronic disease (ACD) is an anaemia that occurs along with chronic inflammatory or neoplastic disorders [9]. The anaemia is mainly due to impaired erythropoiesis, which results in low reticulocyte production.

Disorders and conditions that cause non regenerative anaemias are [19]:

- $\quad$ Reduced Erythropoiesis

- Inflammatory diseases (primarily chronic):

- Infectious: Bacterial, fungal, viral, protozoal, parasitic

- Non infectious

- $\quad$ Renal disease (chronic).

- Diseases causing marrow hypoplasia or aplasia:

- Infectious agents: Bacterial, fungal, viral, protozoal

- Toxicosis: Chemotherapeutic agents, estrogen, bracken fern, phenylbutazone

- Irradiation: Whole body or environmental

- Marrow neoplasia or replacement: Neoplasia, myelofibrosis, osteopetrosis.

- Diseases causing selective erythroid hypoplasia or aplasia:

- $\quad$ Pure red cell aplasia (including immune mediated mechanisms)

- $\quad$ FeLV induced erythroid hypoplasia

- Endocrine: Hypothyroidism, hypoadrenocorticism, hypoandrogenism

- $\quad$ Liver disease or failure (including portosystemic shunts). 
- Ineffective Erythropoiesis:

- $\quad$ FeLV induced erythroid neoplasia

- $\quad$ nutritional: Iron, copper, cobalt, folate, or vitamin B12 deficiency.

- Immune mediated non regenerative anaemia (includes at least some pure red cell aplasias)

- $\quad$ Dyserythropoiesis of English Springer spaniels.

- $\quad$ Congenital dyserythropoiesis of polled Hereford calves.

Further classification is done on the basis of MCV and MCHC as these values are valid and reflect a pathologic state and are used to characterize erythrocytes in an anaemic blood, but abnormal values can also be found when anaemia is not present [19].

MCV and MCHC suggest the type of erythrocytes that is being produced by the marrow, although post production processes can influence the values if severe enough:

- $\quad$ Normocytic - Erythroid cell maturation is not defective.

- Macrocytic - Young erythrocytes are present or erythrocyte maturation is defective.

- Microcytic - Mitosis during erythropoiesis may create smaller cells.

- Normochromic - Haemoglobin synthesis is complete.

- Hypochromic - Haemoglobin synthesis is incomplete (young erythrocytes or defective synthesis).

- Hyperchromic - Erythrocytes were not hyperchromic when produced. Either they lost volume (in vitro or in vivo) or there is an erroneous MCHC:

- Normocytic normochromic anaemias: Most anaemias begin as normocytic normochromic. If this condition persists, then typically disorders that reduce erythropoiesis are suspected. Most anaemias in horses are normocytic normochromic because their marrow release few reticulocytes.
- Macrocytic hypochromic anaemias: Concurrent macrocytosis and hypochromasia support the presence of immature erythrocytes, and thus this anaemia is probably due to blood loss or haemolysis. Dogs with stomatocytosis may have macrocytic hypochromic cells. It is generally due to the regenerative response after blood loss or haemolysis.

- Macrocytic normochromic anaemias: They are common in regenerative anaemias because of blood loss or haemolysis. They are sometimes associated with defective erythropoiesis.

Disorders or conditions related to macrocytic normochromic anaemia are [19]:

- $\quad$ FeLV-infected cats may have defective erythroid maturation that yields megaloblastic cells with defective DNA synthesis and thus decreased mitoses; megaloblastic cells mature to macrocytes.

- $\quad$ Folic acid and cobalamin (vitamin $\mathrm{B}_{12}$ ) deficiencies cause defective nucleic acid metabolism that could cause macrocytosis (possible, but rarely documented). Cattle that graze a cobalt deficient pasture may have a normocytic or macrocytic anaemia due to a cobalamin deficiency.

- $\quad$ Poodles with the poodle marrow dyscrasia will have a macrocytosis and may have an anaemia due to another pathologic process.

- $\quad$ Erythroleukemia.

- Congenital dyserythropoiesis and progressive alopecia of polled Hereford calves.

- Microcytic hypochromic anaemias: Microcytosis and Hyperchromasia maybe due to hepatic failure (rarely; more likely microcytic normochromic) or defective haemoglobin synthesis caused by the following:

- Iron deficiency.

- $\quad$ copper deficiency in dogs may cause a microcytic hypochromic anaemia. However, an experimental copper deficiency in dogs produced a normocytic normochromic anaemia.

- Potentially, vitamin $B_{6}$ (pyridoxine) deficiency. 
- Microcytic normochromic anaemias: This condition can be caused due the following causes:

- Iron deficiency (early or mild)- Prior to causing a microcytic hypochromic anaemia, iron deficiency may produce a microcytic normochromic anaemia, but the $\mathrm{MCH}$ is decreased.

- Hepatic failure due to hepatic disease or portosystemic shunt- The cause of microcytosis is not known, but the data suggest a defect in iron transport to erythrocyte precursors. MCH is decreased but the MCHC typically remains within reference interval (WRI).

- Dyserythropoiesis in English Springer Spaniels.

- $\quad$ Some healthy Akitas and Shibas have lower MCV values than do dogs of other breeds.

- Normocytic hypochromic anaemias: These animals are uncommon. they can be found when erythrocytes are hypochromic (because of immaturity or iron deficiency) and the MCV has not changed enough to be outside of reference interval.

- Macrocytic hyperchromic anaemias: Typically, the MCHC is falsely increased as this condition is quite rare.

- Normocytic hyperchromic anaemias: Typically, the MCHC is falsely increased as this condition is quite rare.

- Microcytic hyperchromic anaemias: Falsely low MCV and MCHC may be produced when erythrocytes are in hypo-osmolal plasma. Erythrocytes adjust in vivo to the hypo-osmolal environment caused by hyponatremia and hypochloraemia by having decreased cytoplasmic osmolality. When placed in a diluent prior to counting, osmosis results in water leaving the erythrocytes and thus decreasing volume of erythrocytes.

\section{Hyperchromasia [9]}

This is the increased staining of the erythrocyte due to increased thickness of erythrocyte. Hyperchromasia of red blood cells has been described in many red blood cell membranopathies, especially HS. In addition to RBC membranopathies, erythrocytic hyperchromasia can also be caused by autoimmune haemolytic anaemia (AIHA), acute oxidant injury, micro- and macroangiopathic haemolytic anaemias, haemolytic transfusion reactions, thermal injuries, liver disease, clostridial sepsis, zinc toxicity, poisoning by certain snake, spider and hymenoptera venoms, severe hypophosphatemia, hypersplenism7, artificial heart valves, massive myocardial infarction and cytotoxic treatment.

\section{Hypochromasia [9]}

This is the decreased staining intensity of erythrocytes which may be either due to decreased thickness of erythrocyte or decreased haemoglobin content. It can be seen in case of iron deficiency in the body, poisoning especially lead poisoning, excessive blood loss maybe in case of an injury or leukaemia, deficiency of Vitamin B6, ulcers, piles, gastric bleeding, hermoloids and gene mutations.

\section{Polychromasia [9]}

In this case, the erythrocytes stain with many colours, i.e., red, blue and intermediate colours. This usually happens when red blood cells are immature because they were released too early from the bone marrow.

\section{Increased ESR}

This condition can be used to interpret acute generalized infections, pericarditis, peritonitis, or malignant neoplasms. Some specific diseases also show this feature, like, Canine Distemper, Leptospirosis, pyometra, filariasis or bone fractures in dogs and Strongyloides infection or Equine Infectious Anaemia in horses.

\section{Decreased ESR}

This condition is generally seen along with liver diseases (low plasma protein), poor nutrition and increased immature RBCs in dogs and in hyperglobulinaemia in horses.

\section{Anisocytosis}

This refers to variation in volumes of erythrocytes. It can be caused by macrocytes (erythrocytes that have increased volume), microcytes (erythrocytes that have decreased volume), or both. Because of their decreased diameters, spherocytes may produce apparent anisocytosis even if the spherocyte volumes are not decreased. RDW is an automated measure of anisocytosis based on volumes, not on the microscopic assessment of the cell diameters. 


\section{Leukocytes (WBC)}

Total leukocyte count (TLC)

TLC is defined as the number of leukocytes or white blood cells present in a given volume of blood. Leukocytes mediate the defence mechanism of the body and are expressed in thousand $\left(10^{9}\right)$ cells per microlitre of blood.

The normal values of TLC in different species are:

\begin{tabular}{|c|c|}
\hline [5] Species & TLC $(\mathbf{m i l l i o n} / \boldsymbol{\mu l})$ \\
\hline Cattle/Buffalo & $5-10$ \\
\hline Horse & $6-10.4$ \\
\hline Sheep & $9-15$ \\
\hline Goat & $8-18$ \\
\hline Dog & $4.95-7.87$ \\
\hline Cat & $5-10$ \\
\hline Pig & $5-8$ \\
\hline Poultry & 1.7 \\
\hline
\end{tabular}

Table 9

Differential leukocyte count (DLC)

The differential leucocyte count (DLC) measures the percentage of each type of WBC present in the blood, i.e. the number of various leukocyte types per hundred WBCs counted [11]. A differential count also can detect immature WBCs and abnormalities.

\begin{tabular}{|c|c|c|c|c|c|}
\hline $\begin{array}{c}\text { [5] Spe- } \\
\text { cies }\end{array}$ & $\begin{array}{c}\text { Neutro- } \\
\text { phils \% }\end{array}$ & $\begin{array}{c}\text { Lympho- } \\
\text { cytes \% }\end{array}$ & $\begin{array}{c}\text { Mono- } \\
\text { cytes \% }\end{array}$ & $\begin{array}{c}\text { Eosino- } \\
\text { phils \% }\end{array}$ & $\begin{array}{c}\text { Baso- } \\
\text { phils \% }\end{array}$ \\
\hline $\begin{array}{c}\text { Cattle/ } \\
\text { Buffalo }\end{array}$ & $15-33$ & $52-63$ & $0-8$ & $0-20$ & $0-2$ \\
\hline Horse & $52-70$ & $21-42$ & $0-6$ & $6-7$ & $0-2$ \\
\hline Sheep & $10-50$ & $40-75$ & $0-6$ & $0-10$ & $0-3$ \\
\hline Goat & $30-48$ & $50-70$ & $0-4$ & $1-8$ & $0-1$ \\
\hline Dog & $58-85$ & $8-21$ & $2-10$ & $0-9$ & $0-1$ \\
\hline Cat & $45-64$ & $27-36$ & $0-5$ & $0-4$ & $0-1$ \\
\hline Pig & $20-70$ & $35-75$ & $0-10$ & $0-15$ & $0-3$ \\
\hline Poultry & $25-30$ & $56-60$ & $5-10$ & $3-8$ & $1-4$ \\
\hline
\end{tabular}

Table 10

\section{Absolute leukocyte count (ALC)}

It is the actual measure of various leukocyte types per unit volume of the blood. It is expressed in no. of cells per microlitre of blood.

\begin{tabular}{|c|c|c|c|c|c|}
\hline [5] Species & \begin{tabular}{|c} 
Neutro- \\
phils \\
$(\mathbf{x 1 0} /$ \\
$\mu l)$ \\
\end{tabular} & $\begin{array}{c}\text { Lympho- } \\
\text { cytes } \\
\left(\mathrm{x} 10^{9} /\right. \\
\mu \mathrm{l})\end{array}$ & $\begin{array}{c}\text { Mono- } \\
\text { cytes } \\
\left(\times 10^{9} /\right. \\
\mu l)\end{array}$ & $\begin{array}{c}\text { Eosino- } \\
\text { phils } \\
\left(\times 10^{9} / \mu \mathrm{l}\right)\end{array}$ & $\begin{array}{c}\text { Baso- } \\
\text { phils } \\
\left(\times 10^{9} /\right. \\
\mu \mathrm{l})\end{array}$ \\
\hline $\begin{array}{l}\text { Cattle/Buf- } \\
\text { falo }\end{array}$ & $0.6-4.0$ & $52-63$ & $0-8$ & $0-20$ & $0-2$ \\
\hline Horse & $2.9-8.5$ & $21-42$ & $0-6$ & $6-7$ & $0-2$ \\
\hline Sheep & $10-50$ & $40-75$ & $0-6$ & $0-10$ & $0-3$ \\
\hline Goat & $30-48$ & $50-70$ & $0-4$ & $1-8$ & $0-1$ \\
\hline Dog & $2.9-12.0$ & $0.4-2.9$ & $0.1-1.4$ & $0-1.3$ & $0-0.1$ \\
\hline Cat & $2.5-12.5$ & $27-36$ & $0-0.9$ & $0-0.8$ & $0-0.2$ \\
\hline Pig & $20-70$ & $35-75$ & $0-10$ & $0-15$ & $0-3$ \\
\hline Poultry & $\begin{array}{c}29.5- \\
37.3\end{array}$ & $48.9-58.4$ & $9.7-10.2$ & 1.7 & $0.7-2$ \\
\hline
\end{tabular}

Table 11

Disorders related to deviation in normal leukocytic parameters

A deviation from the normal level of these parameters can indicate a number of disorders like.

\section{Disorders related to neutrophils [9]}

Neutrophils are important in defence against bacterial and fungal infections and are the main cells of acute inflammation. Therefore, evaluation of numbers and age of neutrophils is particularly useful in disease assessment.

\section{Neutrophilia}

This condition also called neutrophil leucocytosis or occasionally neutrocytosis) is leucocytosis (elevation of WBC from normal level) of neutrophils, that is, a high number of neutrophils in the blood [12]. In very severe inflammations, sequestration of the entire circulating neutrophil pool, and most of the marrow postmitotic maturation and storage pool, may occur at the site of inflammation [9]. The circulating band and metamyelocyte neutrophil numbers may be similar to or greater than mature neutrophils. When there is increase in immature neutrophils (above 6\% of TLC), this is termed as Shift to left, whereas, increase in number of mature neutrophils, it is referred as Shift to right.

Neutrophilia can be seen in inflammations (bacterial, rickettsial, mycotic and parasitic.), trauma, neoplasia, intoxication, surgical procedures, hyperadrenocorticism and in metabolic and endocrine disorders [13]. 
Disorders and conditions that cause neutrophilia are [19]:

- Inflammatory neutrophilia:

- Infections: Bacterial, fungal, viral, protozoal

- Immune haemolytic anaemia

- Necrosis: Haemolysis, hemorrhage, infarcts, burns, neoplasia, sterile inflammation

- $\quad$ Sterile foreign body.

- $\quad$ Steroid neutrophilia:

- $\quad$ Stress (physical or neurogenic)

- Hyperadrenocorticism

- Glucocorticoid therapy

- Adrenocorticotropic hormone administration.

- $\quad$ Physiological (shift) neutrophilia:

- $\quad$ Fight-or-flight response: Excitement, fright, pain, exercise, anxiety

- Catecholamine injections: Epinephrine or norepinephrine.

- Chronic neutrophilic leukaemia

- Paraneoplastic neutrophilia

- $\quad$ Others or unknown mechanisms:

- Neutrophilia of leukocyte adhesion deficiency

- G-CSF administration

- $\quad$ Estrogen toxicosis (early).

\section{Neutropenia}

This term refers to a reduction in the number of circulating neutrophils in the body. It occurs from depletion of the circulating and marrow post-mitotic maturation and storage pools in animals with severe infections [9]. This condition is seen in shock condition, bone marrow depression, overwhelming bacterial/viral infections and in deficiency of Vitamin $\mathrm{B}_{12}$ and folic acid.
Disorders and conditions that cause neutropenia are [19]:

- Inflammation:

- Overwhelming bacterial infections: Equine salmonellosis

- Some viral infections: Canine and feline parvovirus, equine influenza

- Variety of inflammatory states in cattle: Mastitis, pneumonia.

- Peripheral destruction:

- Immune-mediated neutropenia

- Hemophagocytic syndromes.

- Granulocytic hypoplasia:

- Infectious: Parvovirus (dogs and cats) FeLV, Toxoplasma, Ehrlichia

- Neoplastic: Primary or metastatic.

- Toxic:

- Predictable: Estrogen, chemotherapeutic drugs, chloramphenicol (cats)

- Idiosyncratic: Phenylbutazone, bracken fern, griseofulvin.

- Marrow necrosis

- Myelofibrosis.

- Ineffective production:

- Immune-mediated neutropenia

- Diphenylhydantoin and phenylbutazone toxicosis (suspected in animals)

- $\quad$ Chronic idiopathic neutropenia (G- CSF deficiency).

- Cyclic haematopoiesis:

- $\quad$ Cyclic haematopoiesis of grey collies

- $\quad$ Cyclic haematopoiesis associated with FeLV. 
Disorders related to lymphocytes [9]

Lymphocytes are a type of white blood cell that play several roles in the immune system, including protection against bacteria, viruses, fungi, and parasites [14].

There are three types of lymphocytes: B cells (B lymphocytes), T cells (T lymphocytes), Natural killer cells (NK Cells).

\section{Lymphocytosis}

This term refers to an increase in the absolute number of circulating lymphocytes in the body. All three types can be increased in response to infections or cancer [9]. However, in some cases only a specific type of lymphocyte is increased.

This condition is seen in case of viral infections, autoimmune diseases, hypoadrenocorticism, lymphocytic leukaemia and lymphadenitis / lymphangitis [9]. However, this condition is physiological in calves and kittens.

Disorders and conditions that cause lymphocytosis are [19]:

- Chronic inflammation:

- $\quad$ Bacterial infections, specially anaplasmal (e.g., Ehrlichia canis)

- $\quad$ Fungal infections, primarily systemic

- Viral infections: FeLV, BLV, equine infectious anaemia virus

- Protozoal infections, especially babesial and theilerial.

- $\quad$ Physiological (shift) lymphocytosis:

- $\quad$ Fight- or- flight response: Excitement, fright, pain, exercise, anxiety

- Catecholamine injections: Epinephrine or norepinephrine.

- Lymphoproliferative disorders:

- Lymphoma (BLV, FeLV, and idiopathic), leukemic phase

- $\quad$ Lymphoid leukemia

- $\quad$ Persistent lymphocytosis of cattle (BLV).

- Hypoadrenocorticism.
Lymphopenia

When there is decrease in absolute count of lymphocytes, the condition is called lymphopenia, often seen in chronic enteric diseases, hyperadrenocorticism, radiation exposures and in some viral infections like Canine distemper, Infectious canine hepatitis, Bovine viral diarrhoea, Hog cholera.

Disorders and conditions that cause lymphopenia are [19]:

- Acute inflammation:

- Acute bacterial infections

- $\quad$ Acute viral infections

- Endotoxemia.

- $\quad$ Steroids

- Depletion:

- $\quad$ Lymphoid effusion: Chylothorax, and feline cardiomyopathy

- Loss of lymph: Alimentary lymphoma, enteric neoplasms, granulomatous enteritis, paratuberculosis, protein- losing enteropathy, lymphangiectasia, ulcerative enteritis.

- Lymphoid hypoplasia or aplasia:

- Immunosuppressive drugs or whole-body irradiation

- Destruction of lymphoid tissues: Multicentric lymphoma, generalised lymphadenitis

- Combined immunodeficiency of horses (Arabian and Appaloosa) and dogs (basset hound, Cardigan Welsh Corgi, and Jack Russell terrier)

- $\quad$ Thymic aplasia of black- pied Danish title.

\section{Disorders related to monocytes [9]}

Monocytes are produced in bone marrow and then are transported in the blood to tissues and body cavities [9]. Here, they rapidly differentiate into macrophages. Monocytes remain in the bone marrow for about 24 hours before entering the peripheral blood. 


\section{Monocytosis}

This term refers to increase in absolute count of monocytes in blood [9]. This condition in animals indicates an increased demand for macrophages in the tissues. This is particularly seen in haemolytic anaemias. For example, blood smears from cat, with severe Haemobartonella felis infections or dogs with immune-mediated haemolytic anaemia usually have numerous monocytes in route to the spleen. Monocytosis is also present in chronic suppurative inflammatory states, such as pyometra in dogs, and traumatic pericarditis in cattle. Monocytosis occurs in dogs and cattle following administration of glucocorticoid drugs.

Disorders and conditions that cause monocytosis are [19]:

- Inflammation:

- Infections: Bacterial (including anaplasmal and rickettsial), fungal, protozoal

- Necrosis: Haemolysis, hemorrhage, neoplasia, infarction, trauma.

- Steroids:

- $\quad$ Stress (physical or neurogenic)

- Hyperadrenocorticism

- Glucocorticoid therapy

- Adrenocorticotropic hormone administration.

- Neoplasia: Monocytic leukemia

- $\quad$ Secondary to immune mediated neutropenia

- $\quad$ Cyclic hematopoiesis

- G-CSF administration.

\section{Monocytopenia [15]}

A low number of monocytes in the blood can be caused by anything that decreases the overall white blood cell count, such as a bloodstream infection, chemotherapy, or a bone marrow disorder.
This condition is difficult to document because healthy domestic mammals may have relatively few blood monocytes. Monocytopenia is not considered a diagnostic problem.

\section{MonoMAC syndrome [15]}

A rare genetic disorder that affects the bone marrow, causes a very low monocyte count and also decreases the number of certain kinds of lymphocytes. The disorder increases the risk of infection with certain organisms, including a group of bacteria known as Mycobacterium avium complex (MAC) that are related to tuberculosis, human papillomavirus (HPV), and certain fungi. People are also at risk of developing some types of leukaemia.

\section{Disorders related to eosinophils [9]}

Infiltration of tissues by eosinophils is somewhat independent of other leukocytes. Three cytokines, IL-3, IL-5, and GM-CSF, are particularly important in regulating the production of eosinophils. Eosinophils are attracted to tissues as a part of T lymphocyte-mediated immune response to certain antigens, mainly allergens and those of helminths.

\section{Eosinophilia}

The most common causes of a high number of eosinophils, also called hyper-eosinophilia are [16]:

- $\quad$ Allergic disorders

- Infections by parasites

- $\quad$ Certain cancers.

Allergic disorders, including drug sensitivities, asthma, allergic rhinitis, and atopic dermatitis, often increase the number of eosinophils [16]. Many parasites, particularly ones that invade tissue, cause eosinophilia. Cancers that cause eosinophilia include Hodgkin lymphoma, leukaemia, and certain myeloproliferative neoplasms.

Disorders and conditions that cause eosinophilia are [19]:

- Hypersensitivity (allergic) disorders:

- $\quad$ Flea bite dermatitis 
- Hypersensitivity to Staphylococcus or streptococcal proteins

- $\quad$ Milk allergy in ruminant

- $\quad$ Asthma and eosinophilic respiratory disorders.

- Parasitism:

- $\quad$ Ectoparasites

- Heartworms

- $\quad$ Tissue nematodes, trematodes, and protozoa:

- Dogs: Dirofilaria, Acanthocheilonema (Dipetalonema), Spirocerca, Strongyloids, Trichuris, and Paragonimus infections; larval migration of hookworms and round worms; Habronema

- $\quad$ Cats: Paragonimus, Aeleurostrongylus

- Horses: Strongyloides

- $\quad$ Cattle: Sarcocystis.

- Mast cell degranulation caused by inflammation: Cutaneous, respiratory, intestinal, genital, urinary

- Hypoadrenocorticism

- Idiopathic eosinophilic conditions:

- Dog: Eosinophilic myositis, eosinophilic gastroenteritis, eosinophilic panosteitis, eosinophilic pneumonitis, eosinophilic granuloma complex in Siberian Huskies

- Cat: Eosinophilic granuloma complex, eosinophilic enteritis, hypereosinophilic syndrome

- Paraneoplastic eosinophilic (including mast cell neoplasms).

- $\quad$ Eosinophilic leukemia.

\section{Eosinopenia}

A low number of eosinophils in the blood can occur with Cushing Syndrome, bloodstream infections (sepsis), and treatment with corticosteroids. However, a low number of eosinophils does not usually cause problems because other parts of the immune system compensate adequately [16].

Disorders and conditions that cause eosinopenia are [19]:

- $\quad$ Steroids

- Acute Inflammation

- Diseases causing a hyperplastic to aplastic marrow.

\section{Disorders related to basophils [9]}

Like eosinophils, basophils and mast cells are specialized effector cells of the immune system and they both play both pro-inflammatory and immunomodulatory roles in allergic disorders.

\section{Basophilia}

Basophilia or increase in basophils is rare in animals, but is at times seen along with eosinophilia [9]. This is because basophils and eosinophils share many immuno-phenotypic properties. Sometimes basophils and mast cells are seen with eosinophils in tracheal washes of dogs and horses in intermittent chronic bronchitis, probably hypersensitivity disorders. The same is seen in tracheal washes from dogs containing Oslerus osleri larvae. Basophilia is also seen in hypothyroidism, basophilic leukaemia and hyperadrenocorticism in dogs.

Disorders and conditions that cause basophilia are [19]:

- $\quad$ Allergic reactions (immediate or delayed)

- $\quad$ Drugs foods, inhalants, and insect stings or bites

- Parasitism

- $\quad$ Fleas

- Gastrointestinal parasites such as nematodes

- Vascular parasites such as Dirofilaria immitis and Acanthocheilonema (Dipetalonema) reconditum

- Neoplasia

- $\quad$ Basophilic leukemia 
- Mast cell neoplasia

- $\quad$ Feline myeloproliferative diseases

- $\quad$ Lymphomatoid granulomatosis

- $\quad$ Essential thrombocythemia

- Polycythaemia vera.

Thrombocytes (Platelets)

\section{Platelet count}

Platelets, also called thrombocytes, are tiny fragments of cells that are essential for normal blood clotting [17]. They are formed from very large cells called megakaryocytes in the bone marrow and are released into the blood to circulate. The platelet count is a test that determines the number of platelets in your sample of blood.

\begin{tabular}{|c|c|}
\hline [5] Species & Thrombocytes $\left(\mathbf{1 0}^{\mathbf{9}} / \boldsymbol{\mu l}\right)$ \\
\hline Cattle/Buffalo & $100-800$ \\
\hline Horse & $117-256$ \\
\hline Sheep & $800-1100$ \\
\hline Goat & $300-600$ \\
\hline Dog & $211-621$ \\
\hline Cat & $300-800$ \\
\hline Pig & $200-500$ \\
\hline
\end{tabular}

Table 12

\section{Mean platelet volume (MPV)}

An MPV blood test measures the average size of the platelets present in blood [19]. The test can help diagnose bleeding disorders and diseases of the bone marrow.

\begin{tabular}{|c|c|}
\hline [5] Species & MPV (fL) \\
\hline Cattle/Buffalo & $3.5-6.5$ \\
\hline Horse & $4-6$ \\
\hline Sheep & $3.6-6.5$ \\
\hline Goat & $3.7-7.1$ \\
\hline Dog & $6.1-10.1$ \\
\hline Cat & $12-18$ \\
\hline Pig & $6.7-11.7$ \\
\hline
\end{tabular}

Table 13
Disorders related to deviation in normal thrombocytic parameters

A deviation from the normal level of these parameters can indicate a number of disorders like.

\section{Thrombocytosis [9]}

Thrombocytosis is an increase in the number of blood platelets [9]. Platelet numbers fluctuate in the blood of animals and increase greatly in response to most haemopoietic stimuli.

Disorders and conditions that cause thrombocytosis are [19]:

- Hemic neoplasia (clonal thrombocytosis):

- Primary (essential) thrombocythemia and other chronic myeloproliferative diseases

- $\quad$ Acute megakaryoblastic leukemia.

- $\quad$ Reactive thrombocytosis (secondary, non-clonal):

- Redistribution:

- $\quad$ Exercise

- $\quad$ Epinephrine.

- Increased production:

- Inflammation: Infection, immune mediated, surgery, trauma

- Nonhemic neoplasia

- Iron deficiency

- $\quad$ Vinca alkaloids (vincristine, vinblastine)

- Recovery from thrombocytopenia (rebound): Withdrawal of myelosuppression, recovery from IMT

- $\quad$ Splenectomy (post)

- Blood loss. 


\section{Thrombocytopaenia [9]}

Thrombocytopaenia is a decrease in the number of blood platelets [9]. It is an important disorder in animals. Thrombocytopaenia, like neutropenia or anaemia, is due to premature cellular destruction or defective production. This condition is common in case of Infectious Canine Hepatitis and Swine Fever (Hog Cholera) and also as a result of infection of platelets by rickettsial organism Ehrlichia platys in dogs. Haemorrhages due to thrombocytopaenia helps in the clinical detection of many disorders like Oestrogen toxicity in dogs and Bracken fern poisoning in cattle.

Disorders and conditions that cause thrombocytopenia are [19]:

- Abnormal platelet distribution (sequestration): Splenomegaly, severe hypothermia.

- Decreased platelet production (myelosuppression of one to all hemic cell lines):

- Acquired amegakaryocytic thrombocytopenia

- $\quad$ Drugs (toxicants)

- $\quad$ Predictable: Chemotherapeutic agents, oestrogen in dogs (exogenous, endogenous), bracken fern poisoning (ruminant), trichothecene mycotoxins

- Idiosyncratic: Phenylbutazone, meclofenamic acid, trimethoprim-sulfamethoxazole, griseofulvin

- Infectious (usually multifactorial)

- Irradiation (whole body or extensive): Prior to autologous marrow transplantation

- Marrow replacement: Bone marrow neoplasia (primary hemic or metastatic), myelofibrosis, osteopetrosis

- $\quad$ Megakaryocytic leukemia

- Myelonecrosis: Infections, neoplasia, toxicants.

- Decreased platelet survival (accelerated platelet destruction and consumption):
- Immunologic:

- $\quad$ Primary (idiopathic) IMT

- Secondary IMT:

- $\quad$ Drug induced: Gold salts, sulfonamides

- Infectious (usually multifactorial)

- Neonatal alloimmune thrombocytopenia

- Neoplasia (usually multifactorial)

- Posttransfusion purpura

- $\quad$ Systemic immune-mediated disease: SLE, Evan's syndrome.

- Nonimmunologic:

- Blood loss, acute and severe: Anticoagulant rodenticides

- $\quad$ Platelet activation with accelerated consumption or utilization

- Localised intravascular coagulation: Hemangiosarcoma, hemorrhage, thrombosis

- $\quad$ DIC: Envenomation, hepatic disease, infections, massive necrosis, pancreatitis, neoplasia, overheating, septicemia

- Drugs: Protamine sulfate

- $\quad$ Envenomation (without DIC)

- Vasculitis/endocarditis: Rocky Mountain spotted fever canine herpesvirus infection, haemolytic uremic syndrome, dirofilariasis, angiostrongylosis, bacterial endocarditis, haemolytic uremic syndrome (cutaneous and renal glomerulus vasculopathy of greyhounds).

- Haemodilution: Massive infusion with colloids, platelet- poor blood products

- Idiopathic mechanism or multifactorial (decreased production and decreased survival): 
- Idiopathic thrombocytopenia of cavalier King Charles spaniel

- Infections: Anaplasma platys, Anaplasma phagocytocytophilum, Babesia canis, Babesia gibsoni, bovine viral diarrhoea virus, canine distemper virus, canine parvovirus, Cytauxzoon felis, Ehrlichia spp., equine infectious anaemia virus, feline leukaemia virus, feline immunodeficiency virus, Histoplasma capsulatum, Leishmania spp., Leptospira spp., Theileria spp.

- $\quad$ Endotoxemia

- Neoplasia: Carcinomas, hemangiosarcoma and other sarcomas, lymphoma, leukaemias

- $\quad$ Drugs

- Hypophosphatemia associated with hyperalimentation

- Anaphylaxis.

\section{Platelet function disorders [9]}

These disorders are characterized by spontaneous occurrence of petechiae and mucosal bleeding, and life-threatening haemorrhages following surgery, despite normal platelet counts [9]. These functional defects may be congenital or acquired. They occur from defective platelet secretion, adhesion, or aggregation. The ability of the platelets to adhere to sub-endothelial collagen in spite of rapid blood flow in arterioles is mediated by von Willebrand cyto-adhesive protein. Congenital absence or decrease in this protein results in von Willebrand's disease.

Platelet secretion, adhesion, aggregation and prostaglandin metabolism are damaged in uraemia, and are responsible for the mucosal haemorrhage that occurs in animals with renal failure. Platelet aggregates are mostly unstable and disintegrate before stabilization of the platelet plug by fibrinogen. A disorder of bleeding in calf is characterized by an inadequate content of adenosine diphosphate (ADP) in platelets. ADP released following platelet activation is an important activating agent for nearby platelets, thus facilitating platelet aggregation.

\section{Coagulation disorders [9]}

Platelet disorders result in bleeding from mucosal surfaces because of inadequate plugging at sites of endothelial damage [9]. Disorders of coagulation, also known as coagulopathies, result from failure of fibrin synthesis within platelet aggregates. This causes inadequate stabilization of the platelet plug. Prolonged bleeding then occurs after temporary occlusion of the injured vessel wall by the unstable platelet plug.

The above-mentioned medical conditions are just a glimpse of the vast world of disorders that can be interpreted by just a simple blood test. As the blood is flowing throughout the body, its elements respond differently and thus this elementary test is very basic and gives a bigger picture of any medical condition present in any organ of the body.

\section{How does labs analyse samples for haematology (CBC)?}

From times when analysis blood parameters like RBC count, WBC count, Platelet count, Haemoglobin estimation is totally done by manual methods consuming lot of time and human resource scenario is entirely flipped these days. Automatic analysers totally replaced manual methods for $\mathrm{CBC}$ determination. The technology is need of the hour to support a vast system of health management which can never be only relied only on manual methods. In addition to work-speed the specificity and sensitivity of the Automatic analysers are really impressive.

\section{Principle behind automatic analysers [20]}

Various technological solutions and preparations are used to detect various cells in blood sample. These solutions actually impart different fluorescent staining characteristics to cells and light emitted by them is captured. Also enzyme characteristics like presence of myeloperoxidase in neutrophils is also determined. For determining volumes Coulter principle is used to measure direct current impedance. All these data ultimately forms different histograms. These histograms are analysed by software's to produce results in form of Clinical parameters. The technology used here is referred to VCS (Volume, Conductivity, Light Scatter) technology. Analysers that are much popular these days are Flow cytometry analysers, Electric impedance analysers and Laser scattering analysers.

\section{Recent advancements and future prospects in veterinary dis- ease diagnosis}

One of the recent advancement in Human Medicine is invention of a device that can tell patients their blood parameters at there home. This device is under FDA Class 2 clearance process and reported from Athelas by a diagnostic test developer based in Mountain View, Calif [21]. The claim is that by using a single drop of blood, loading to test strip can get results in 60 seconds. This is 
minimally invasive procedure and can be easily done by patients at their convenience. The colossal advantage of this technique is for patients that need regular monitoring like those suffering from chronic diseases like kidney disease and Cancer. Also these devices are connected to mobile phones and also communicate simultaneously to doctors which can help them to make follow up. This aid is really a want in Veterinary Medicine too as it would be of utmost importance for farmers living in rural areas with minimal facilities of labs are present and also the undue stress to take their animals long distances for sample collection and testing can be avoided. Also pet owners will be really benefitted if there is need for regular monitoring of parameters which eventually provide better medical management. Being minimally invasive that is a drop of blood make it really a choice for animals that otherwise need much restraint.

\section{Conclusion}

Summing up, it can be inferred that above mentioned plethora of haematological aspects are just a glimpse of the vast world of interpretations that can be made by just a simple blood test like $\mathrm{CBC}$. As the blood is flowing throughout the body, its elements respond differently and thus this elementary test gives a practitioner insights about gross aspect of underlying medical condition present in the body. Early diagnosis and accurate diagnosis are the two basic aims when dealing with any medical case for a practitioner and Complete Blood Count profile do justice to it in many celebrated aspects.

\section{Acknowledgement}

A heartfelt thanks to our teachers cum mentors in CoVsc and AH, DUVASU, Mathura who always enlighten us with their valuable knowledge so that we can develop our approach while framing a manuscript. All gratitude to my junior Shalvi Srivastava who really worked hard to compile this article to it's best. Last but not the least I would like to thank my mother Rani Saxena and my friend Hitik Pratap Chauhan who always motivate me to give my best in every realm of life.

\section{Bibliography}

1. J Flowers Health Institute. "Importance of Medical Diagnosis Before Treatment".

2. MSD Manual. "Introduction to Laboratory Diagnosis of Infectious Disease" (2020).

3. Medical News Today. "What is Differential Diagnosis".
4. Boston University Research Support. "Blood Collection Guidelines".

5. Merck Manual Veterinary Manual. "Haematologic Reference Ranges".

6. Healthline. "What is $\mathrm{MCH}$ and What Do High and Low Values Mean?".

7. Wikipedia. "The Free Encyclopedia: Mean Corpuscular haemoglobin concentration".

8. Medline Plus. RDW (Red Cell Distribution Width).

9. Vegad JL and Swamy Madhu. "A Textbook of Veterinary Systemic Pathology". 2nd ed.Lucknow. ibdc publishers (1998): 265-316

10. MSD Manual. "Erythrocytosis".

11. Healthline. "White Blood Cell Count and Differential".

12. Wikipedia. "The Free Encyclopedia: Neutrophilia".

13. Leonie Ronald., et al. "Heamtology as a diagnostic tool in bovine medicine". Journal of Veterinary Diagnostic Investigation 26.5 (2014): 592-598.

14. MSD Manual. "Lymphocytic Leukocytosis”.

15. MSD Manual. "Monocyte Disorders".

16. MSD Manual. "Eosinophilic Disorders".

17. Lab Tests Online. "Platelet Count".

18. Healthline. "Understanding Your MPV Test Results".

19. Steven L Stockham and Michael A Scott. "Fundamentals of Veterinary Clinical Pathology". $2^{\text {nd }}$ ed. Iowa, USA. Blackwell Publishing (2002): 53-223.

20. Technological advances in today's hematology analyzers: how they address common laboratory challenges.

21. "New At-Home CBC Device Enables Complete Blood Testing for Cancer Treatments and Biological/Viral Monitoring". Instruments and Equipment, Laboratory Instruments and Laboratory Equipment, Laboratory Management and Operations, Laboratory Pathology, Laboratory Testing (2018).

\section{Volume 3 Issue 10 October 2021}

(C) All rights are reserved by Harshit Saxena and Shalvi Srivastava. 